\title{
Health-related quality of life in Parkinson's disease: a cross-sectional study focusing on non-motor symptoms
}

\author{
Liis Kadastik-Eerme, Marika Rosenthal, Tiiu Paju, Mari Muldmaa and Pille Taba*
}

\begin{abstract}
Background: The objective of this study was to investigate factors affecting health-related quality of life (HRQL) among Estonian persons with Parkinson's disease (PD).

Methods: 268 persons with PD were evaluated using: the Movement Disorder Society Unified Parkinson's Disease Rating Scale (MDS-UPDRS); the Hoehn and Yahr scale (HY); the Schwab and England Activities of Daily Living scale (SE-ADL); the Beck Depression Inventory (BDI); the Mini Mental State Examination (MMSE); the Parkinson's Disease Questionnaire (PDQ-39). Additional questions on clinical and socio-demographic variables were asked during a semi-structured interview. Predictors of HRQoL were tested using multiple regression analysis.

Results: The main predictors of low HRQoL were depression and motor and non-motor aspects of daily living. $59.9 \%$ of the variation in the PDQ-39 summary index (SI) score was explained by the predictive variables identified in this study. None of the socio-demographic variables (age, gender, urban/rural living, marital status, living alone/ with others, education level) were significant predictors of HRQoL. Prevalence of non-motor Parkinson's symptoms were high (99.6\%); cognitive impairment, sleep and urinary problems were the most common. All non-motor symptoms correlated significantly with low HRQoL, except the features of impulse control disorders (ICDs).

Conclusions: Depression and motor and non-motor daily living experiences were found to be significant and independent factors of low HRQOL in persons with PD. Depression was the strongest determinant of low HRQoL. Our results highlight the importance of recognition and management of non-motor symptoms, as these features had more impact on patients' HRQoL than clinically assessed motor symptoms.
\end{abstract}

Keywords: Depression, Health-related quality of life, Impulse control disorders, MDS-UPDRS, Non-motor symptoms, Multiple regression analysis, Parkinson's disease, PDQ-39

\section{Background}

Parkinson's disease (PD) is one of the most common neurodegenerative diseases worldwide, with a prevalence ranging from 31-201 per 100,000 population [1]. The symptoms of PD encompass motor features such as rigidity, bradykinesia, tremor and postural instability [2], and non-motor symptoms including impairment of olfaction, vision, sleep, salivation, gastric and bowel function, sebaceous gland activity, and mood and cognition [3]. PD diagnosis is based upon the presence of a set of cardinal motor signs; progression is defined by the

\footnotetext{
* Correspondence: Pille.Taba@kliinikum.ee

Department of Neurosurgery and Neurology, Tartu University Hospital, L. Puusepa 8, Tartu 51014, Estonia
}

(c) 2015 Kadastik-Eerme et al. This is an Open Access article distributed under the terms of the Creative Commons Attribution

degree of motor disability; management is primarily directed towards improving motor symptoms $[2,4]$.

Persons with PD are particularly vulnerable to deterioration of health-related quality of life (HRQoL) resulting from significant motor disability and the burden of non-motor symptoms. Assessment of HRQoL of persons with PD is thus of essential importance. Several factors have been reported to be associated with low HRQoL. Among motor symptoms, the most significant determinants affecting HRQoL are disease severity, motor complications, postural instability and gait disorder [5-7]. Among non-motor symptoms, depression, anxiety, cognitive impairment, fatigue, pain, urinary disturbances and sleep problems were found to be the most 
significant determinants of HRQoL [5, 8, 9]. Among other factors, a high levodopa equivalent daily dose and comorbidities were found to be prevalent and independent determinants of HRQoL [6]. Parallel to the clinical features, several socio-demographic factors such as level of education and number of people in a household have been described as independent determinants of HRQoL [5, 7].

Most previous studies however have assessed only a limited number of factors in relation to HRQoL. The impact of certain variables (e.g. rural living, marital status, education, comorbidities) have been infrequently systematically studied. The main aim of our study therefore was to evaluate the impact of a wide range of sociodemographic and clinical factors upon the HRQoL of a cohort of persons with PD in Estonia. We also focused on the non-motor symptoms identified by the new version of the Movement Disorder Society Unified Parkinson's Disease Rating Scale (MDS-UPDRS) Part I, which has recently been adopted and validated [10].

\section{Methods \\ Participants}

A total of 268 persons with PD were recruited for a community-based study by the Department of Neurology and Neurosurgery of Tartu University Hospital, which is one of two regional hospitals in Estonia. The study was based on a PD cohort derived from an epidemiological study primarily aimed at investigating the epidemiology, clinical characteristics and treatment of PD in Estonia. The current study was conducted in the city and district of Tartu between October 2010 and May 2013. Patient selection was made via evaluation of information from hospital records, neurologists and general practitioners, nursing homes, the local PD Society, and the database of the Estonian Health Insurance Fund. Only patients who fulfilled the Queen Square Brain Bank Criteria [2, 11] were included in the study. As this was an epidemiological study, all patients with a confirmed PD diagnosis were enrolled, and no specific exclusion criteria were set. The study was approved by the Research Ethics Committee of the University of Tartu and all patients provided signed informed consent.

\section{Materials and procedure}

The demographic and social data (age, gender, urban/ rural living, marital status, living alone/with others, level of education) and data regarding PD (age at disease onset, disease duration, clinical subtype) and other clinical factors (levodopa equivalent daily dose, duration of levodopa treatment, comorbidities) were recorded using specially developed case report forms. Disease subtypes were based upon the most prevalent symptom during standard neurological examinations: (1) tremor;
(2) bradykinesia-hypokinesia; (3) postural instability and gait disorder.

To evaluate HRQoL, PDQ-39-a validated, diseasespecific quality of life instrument-was used [12]. The higher the PDQ-39 summary index (SI) score, the lower the perception by patients of their quality of life. An Estonian version of the PDQ-39 has been shown as a reliable instrument [13].

Non-motor PD symptoms were identified using Part I of the MDS-UPDRS. In 2008 the Movement Disorder Society (MDS) adopted a new, validated version of the MDS-UPDRS, which included several significant updates in comparison with the previous version, including new non-motor symptoms of PD [10]. The Estonian version of the MDS-UPDRS was translated, validated and officially approved in 2011 by the MDS Translation Program for non-English official versions. The MDSUPDRS Part IA contains questions regarding a number of neuropsychiatric symptoms and is completed by the interviewer; Part IB consists of questions on non-motor symptoms and is answered by the patient or caregiver. Other parts of the MDS-UPDRS were used: Part II regards the motor experiences of daily living as assessed by the patient or caregiver; Part III assesses motor symptoms based on an objective neurological examination; Part IV regards motor complications and is completed by the interviewer.

The clinical stage of a patient's PD was assessed using the Hoehn and Yahr scale (HY) [14] and their level of disability by the Schwab and England Activities of Daily Living Scale (SE-ADL) [15]. The HY scale ranges from 1 (unilateral involvement of the body) to 5 (wheelchair bound or bedridden). The SE-ADL scale ranges from $0 \%$ (completely dependent and bedridden) to $100 \%$ (completely independent). For assessment of depression the Beck Depression Inventory (BDI) was used [16], whereby a score of $\geq 14$ is indicative of depression [17]. Cognitive impairment was measured using the Mini Mental State Examination (MMSE) [18], whereby a score of $\leq 24$ is evidence of cognitive impairment.

\section{Statistical analysis}

PDQ-39 SI measures of HRQoL were calculated according to its scoring algorithm $[12,19]$. Prevalence of nonmotor symptoms was based on scores $\geq 1$, which denoted the presence of a symptom. Student's $t$-test and z-test were applied for comparing socio-demographic and clinical outcomes between men and women. The nonparametric Mann-Whitney and Kruskal-Wallis tests were used to compare PDQ-39 SI scores between the groups. Spearman's rank correlation coefficients were calculated to assess associations between variables. Correlation coefficients were interpreted as very weak $(r=0-0.19)$, 
weak $(r=0.20-0.39)$, moderate $(r=0.40-0.59)$, strong $(r=0.60-0.79)$ or very strong $(r=0.80-1.00)$.

A multiple linear regression analysis-based on a backward elimination approach-was conducted to determine the factors that contribute to HRQoL in persons with PD. PDQ-39 SI was used as a dependent variable. The $\mathrm{R}^{2}$ statistic was used to determine the proportion of variance explained by the predictors. The level of statistical significance was set at $\mathrm{p}<0.05$. Statistical analysis was performed using STATA version 12.0 (StataCorp LP, College Station, TX, USA) and SPSS version 20.0 (IBM Corporation, Armonk, NY, USA).

\section{Results}

\section{Patient characteristics}

Demographic and clinical characteristics of the study population are given in Table 1. Men were slightly younger than women (72.1 and 75.6 years respectively, $\mathrm{p}=0.0014)$, which reflects the shorter life expectancy of men in Estonia. No significant differences in terms of PD onset age, disease duration, HY stage, SE-ADL stage or MMSE performance were found between men and women. The mean BDI depression score was significantly higher in women compared to men (17.1 and 12.3 respectively, $\mathrm{p}=0.0001)$. A significantly higher rate of men compared to women were: married $(71.4 \%$ and $32.5 \%$ respectively, $\mathrm{p}<0.0001)$; lived with their spouse and or children ( $89.5 \%$ and $60.1 \%$ respectively, $\mathrm{p}<0.0001)$. There were more widows among female than male patients $(48.5 \%$ and $17.1 \%$ respectively, $\mathrm{p}=0.02$ ). Women had a higher mean number of comorbidities compared to men (2.23 and 1.86 respectively, $\mathrm{p}=0.0124)$, including a significantly higher rate of cardiovascular diseases $(99.4 \%$ and $89.6 \%$ respectively, $\mathrm{p}<0.0001$ ). Mean scores of PDQ-39 domains are presented in Fig. 1. In persons with PD, mobility (mean score 53.1) was the most negative HRQoL domain and social support (mean score 13.17) the least negative domain.

\section{Prevalence of non-motor symptoms}

Among the 268 patients screened, $99.6 \%$ complained of at least one non-motor symptom. There were a mean number of 6.7 non-motor symptoms (SD 2.5) per patient of the 13 symptoms included in the MDS-UPDRS Part I. The most frequent non-motor symptoms were cognitive impairment, night time sleep disorders, bladder disorders, fatigue, pain, daytime sleepiness and depression. Hallucinations and impulsive compulsive disorders (ICDs) were the most infrequently reported non-motor symptoms. We did not find a statistically significant difference between mean BDI scores for persons with PD and with ICDs, and persons with PD and without ICDs (mean score 19.1 and 15.1, respectively, $\mathrm{p}=0.254$ ). Prevalence of non-motor symptoms is given in Table 2.
Table 1 Characteristics of the patients

\begin{tabular}{|c|c|c|}
\hline Variable & Value & Range \\
\hline Age $^{a}$ & 74.2 yr $( \pm 8.8)$ & $47-96$ \\
\hline Disease onset age ${ }^{a}$ & 66.8 yr $( \pm 10.1)$ & $35-88$ \\
\hline Duration of disease ${ }^{a}$ & $7.6 \mathrm{yr}( \pm 5.9)$ & $1-35$ \\
\hline$H Y \geq 3, n(\%)$ & $164(62.6 \%)$ & \\
\hline SE-ADL $\leq 75 \%, n(\%)$ & $139(53.3 \%)$ & \\
\hline MMSE $\geq 25, \mathrm{n}(\%)$ & $197(76.7 \%)$ & \\
\hline $\mathrm{BDI} \geq 14, \mathrm{n}(\%)$ & $134(52.1 \%)$ & \\
\hline \multicolumn{3}{|l|}{ MDS-UPDRS } \\
\hline MDS-UPDRS Part I & $12.52(6.79)$ & $0-38$ \\
\hline MDS-UPDRS Part II & $18.2(8.4)$ & $1-48$ \\
\hline MDS-UPDRS Part III & $46.3(18.3)$ & $10-106$ \\
\hline MDS-UPDRS Part IV & $1.3(3.4)$ & $0-18$ \\
\hline \multicolumn{3}{|l|}{ Clinical subtypes } \\
\hline Tremor dominant & $118(44 \%)$ & \\
\hline Hypokinetic-rigid dominant & $77(28.7 \%)$ & \\
\hline $\begin{array}{l}\text { Postural instability and gait disturbance } \\
\text { dominant }\end{array}$ & $73(27.3 \%)$ & \\
\hline Levodopa equivalent dose per day ${ }^{a}$ & $427.5 \mathrm{mg}( \pm 231.6)$ & $100-1200$ \\
\hline Duration of levodopa treatment ${ }^{a}$ & 3.9 yr $( \pm 5.03)$ & $0.1-23$ \\
\hline \multicolumn{3}{|l|}{ Marital status, n (\%) } \\
\hline Single & $20(7.4 \%)$ & \\
\hline Married & $128(47.8 \%)$ & \\
\hline Divorced & $23(8.6 \%)$ & \\
\hline Widowed & $97(36.2 \%)$ & \\
\hline \multicolumn{3}{|l|}{ Living status, n (\%) } \\
\hline With a spouse & $137(51,1 \%)$ & \\
\hline With children & $55(20,5 \%)$ & \\
\hline Alone & $66(24.6 \%)$ & \\
\hline Nursery home & 10 (3.8 \%) & \\
\hline \multicolumn{3}{|l|}{ Education, n (\%) } \\
\hline \multicolumn{3}{|l|}{ Primary } \\
\hline Secondary & $104(38.8 \%)$ & \\
\hline \multirow[t]{2}{*}{ Higher } & 95 (35.5 \%) & \\
\hline & 69 (25.7\%) & \\
\hline
\end{tabular}

a Mean (standard deviation)

Abbreviations: HY, Hoehn and Yahr stage; SE-ADL, Schwab and England Activities of Daily Living Scale; MMSE, Mini Mental State Examination; BDI, Beck Depression Inventory; MDS-UPDRS, Movement Disorders Society Unified Parkinson's Disease Rating Scale

\section{PDQ-39 and socio-demographic factors affecting HRQoL}

The results of univariate analysis of socio- demographic and clinical parameters and mean scores of PDQ-39 SI in persons with $\mathrm{PD}$ are shown in Table 3. Emotional well-being and bodily discomfort affected women significantly more than men. Being over 65 years old was associated with lower mobility: being younger was associated 


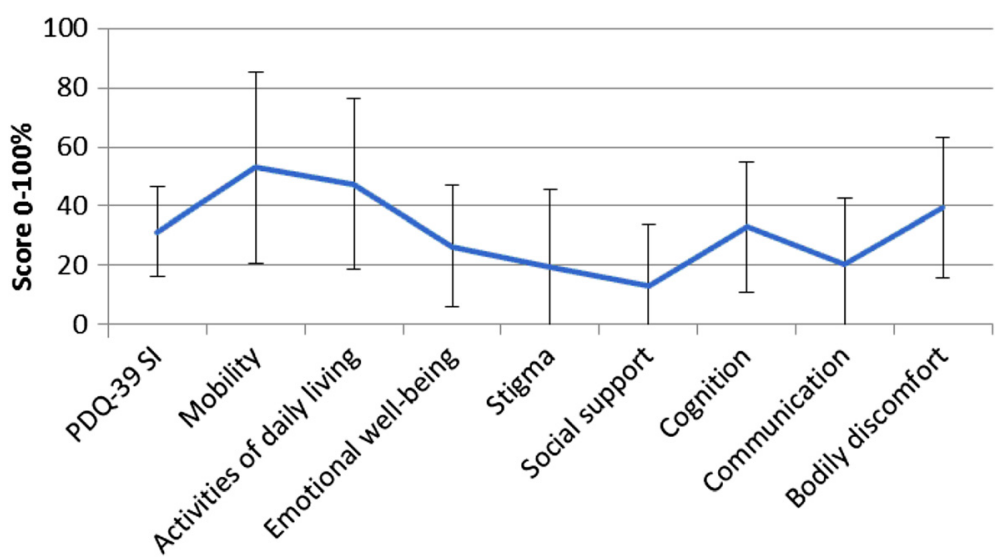

Fig. 1 Mean values of PDQ-39 domains. HRQoL was assessed by using the PDQ-39. PDQ-39 is composed of 39 items grouped in 8 subscales: (1) mobility, (2) activities of daily living, (3) emotional well-being, (4) stigma, (5) social support, (6) cognition, (7) communication, and bodily discomfort. The PDQ-39 SI is an overall score calculated from these domains, with 0 indicating the best HRQoL and 100 the worst

with higher stigmatisation. Marital status was not significantly associated with PDQ-39 scores. Emotional wellbeing was significantly related to living status, i.e. patients living alone had lower emotional well-being compared to patients living with their spouse and/or children. Education did not significantly influence overall HRQoL. People who had undergone no more than primary level education were significantly more affected in the domain of mobility.

\section{PDQ-39 and clinical factors affecting HRQoL}

Overall HRQoL was significantly lower in patients with depression, which had an impact on all the PDQ-39 domains. Longer disease duration, a HY stage $\geq 3$ and SE-ADL $\leq 75 \%$ were associated significantly with lower

Table 2 Prevalence of non-motor symptoms

\begin{tabular}{lll}
\hline Non-motor symptoms & $\mathrm{N}$ & $\%$ \\
\hline Cognitive impairment & 199 & $74.3 \%$ \\
Nighttime sleep problems & 192 & $71.6 \%$ \\
Urinary problems & 192 & $71.6 \%$ \\
Fatigue & 184 & $68.7 \%$ \\
Pain & 172 & $64.2 \%$ \\
Daytime sleepiness & 166 & $61.9 \%$ \\
Depressed mood & 163 & $60.8 \%$ \\
Constipation problems & 156 & $58.2 \%$ \\
Anxious mood & 151 & $56.3 \%$ \\
Lightheadedness on standing & 140 & $52.2 \%$ \\
Apathy & 122 & $45.7 \%$ \\
Hallucinations and psychosis & 37 & $13.8 \%$ \\
ICDs & 21 & $7.8 \%$ \\
\hline Abbretions:
\end{tabular}

Abbreviations: ICDs, impulse control disorders overall HRQoL and affected most of the PDQ-39 domains. Patients with cognitive impairment had lower overall HRQoL and were significantly more adversely affected in the domains of mobility, activities of daily living and communication.

By clinical PD subtype, the group with postural instability and gait disturbance had the lowest overall HRQoL (compared to the tremor and hypokinetic-rigid clinical subtypes). Out of PDQ-39 domains, mobility and activities of daily living had the lowest outcome.

\section{Correlation analysis between PDQ-39 SI and socio-demographic and clinical factors}

PDQ-39 SI correlated significantly with higher MDSUPDRS Part II, BDI and MDS-UPDRS Part IB scores. All non-motor symptoms-except ICDs-correlated significantly with PDQ-39 SI. All correlations between PDQ-39 and the other characteristics of PD are shown in Table 4.

\section{Multiple linear regression model of PDQ-39 SI}

Potential predictors were determined based on the previous correlation analysis. Duration of disease, HY, SE-ADL, MMSE, BDI, MDS-UPDRS Parts IA, IB, II-IV, duration of levodopa treatment, daily dose of levodopa, postural instability and gait disorder or tremor dominated disease types were identified as independent variables using univariate regression analysis. Multiple linear regression analysis was then performed on all the independent variables found during the previous univariate regression analysis and common epidemiological variables, i.e. age and gender. The final model (Table 5) explained $59.9 \%$ (adjusted $\mathrm{R}^{2}$, $\mathrm{p}<0.0001$ ) of the variance of PDQ-39 SI, with BDI, MDS-UPDRS II, MDS-UPDRS Part IB significant 
Table 3 PDQ-39 associations with demographic and clinical features

\begin{tabular}{|c|c|c|}
\hline Variable & PDQ-39 SI ${ }^{a}$ & $p$ value \\
\hline Gender & & 0.066 \\
\hline Male & $29.8(15.5)$ & \\
\hline Female & $32.7(15.1)$ & \\
\hline Living area & & 0.192 \\
\hline Urban & $32.5(15.3)$ & \\
\hline Rural & $29.6(15.1)$ & \\
\hline Marital status & & 0.770 \\
\hline Single & $32.7(17.4)$ & \\
\hline Married & $30.8(15.3)$ & \\
\hline Divorced & $34.2(13.1)$ & \\
\hline Widowed & $31.7(15.4)$ & \\
\hline Living status & & 0.638 \\
\hline With a spouse & $30.6(15.4)$ & \\
\hline With children & $31.5(17.1)$ & \\
\hline Alone & $33.5(13.5)$ & \\
\hline Nursery home & $32.7(15.3)$ & \\
\hline Education & & 0.339 \\
\hline Primary & $33.6(13.6)$ & \\
\hline Secondary & $30.4(15.1)$ & \\
\hline Higher & $30.1(17.6)$ & \\
\hline Clinical subtypes & & $0.0017 ; 1 \mathrm{vs} 3$ \\
\hline Tremor & $28.6(14.9)$ & \\
\hline Hypokinetic-rigid & $30.5(14.4)$ & \\
\hline Postural instability and gait disturbance & $37.3(15.3)$ & \\
\hline Disease duration & & $0.00010 ; 1$ vs 3 \\
\hline$\leq 5 \mathrm{yr}$ & 26.5 (13.0) & \\
\hline $6-10 y r$ & $32.1(16.1)$ & \\
\hline$>10 \mathrm{yr}$ & $38.8(14.8)$ & \\
\hline $\mathrm{HY}$ & & $<0.0001$ \\
\hline$\leq 2.5$ & $24.2(13.9)$ & \\
\hline$\geq 3$ & 35.7 (14.6) & \\
\hline SE-ADL & & $<0.0001$ \\
\hline$\geq 80$ & $25.0(14.4)$ & \\
\hline$\leq 75$ & $37.0(14.0)$ & \\
\hline MMSE & & 0.0037 \\
\hline$\geq 25$ & $29.9(14.9)$ & \\
\hline$\leq 24$ & 35.9 (14.6) & \\
\hline BDI & & $<0.0001$ \\
\hline$<14$ & $23.0(11.9)$ & \\
\hline$\geq 14$ & $39.2(14.1)$ & \\
\hline
\end{tabular}

${ }^{a}$ Mean (standard deviation)

Abbrevations: HY, Hoehn and Yahr stage; SE-ADL, Schwab and England Activities of Daily Living Scale; MMSE, Mini Mental State Examination; BDI, Beck Depression Inventory
Table 4 Spearman's correlation analysis of clinical variables and PDQ-39 SI scores

\begin{tabular}{|c|c|c|}
\hline Variable & Coefficient & $\mathrm{p}$ value \\
\hline Disease duration & 0.30 & $<0.0001$ \\
\hline \multicolumn{3}{|l|}{ Clinical subtypes } \\
\hline Tremor dominant & -0.17 & 0.006 \\
\hline Hypokinetic-rigid dominant & -0.05 & 0.449 \\
\hline Postural instability and gait disturbance dominant & 0.23 & $<0.0001$ \\
\hline HY & 0.51 & $<0.0001$ \\
\hline SE-ADL & -0.51 & $<0.0001$ \\
\hline MMSE & -0.25 & 0.0001 \\
\hline BDI & 0.63 & $<0.0001$ \\
\hline Daily dose of levodopa & 0.32 & $<0.0001$ \\
\hline Duration of levodopa treatment & 0.23 & 0.0001 \\
\hline Number of comorbidities & 0.07 & 0.5041 \\
\hline $\begin{array}{l}\text { MDS-UPDRS Part I - Non-motor experiences } \\
\text { of daily living }\end{array}$ & 0.62 & $<0.0001$ \\
\hline MDS-UPDRS Part IA & 0.51 & $<0.0001$ \\
\hline MDS-UPDRS Part IB & 0.61 & $<0.0001$ \\
\hline Cognitive impairment & 0.38 & $<0.0001$ \\
\hline Hallucinacions and psychosis & 0.24 & 0.0001 \\
\hline Depressed mood & 0.35 & $<0.0001$ \\
\hline Anxious mood & 0.29 & $<0.0001$ \\
\hline Apathy & 0.33 & $<0.0001$ \\
\hline ICDs & 0.07 & 0.2490 \\
\hline Nighttime sleep problems & 0.29 & $<0.0001$ \\
\hline Daytime sleepiness & 0.35 & $<0.0001$ \\
\hline Pain and other sensations & 0.38 & $<0.0001$ \\
\hline Urinary problems & 0.30 & $<0.0001$ \\
\hline Constipation problems & 0.13 & 0.0281 \\
\hline Lightheadedness on standing & 0.34 & $<0.0001$ \\
\hline Fatigue & 0.44 & $<0.0001$ \\
\hline $\begin{array}{l}\text { MDS-UPDRS Part II - Motor experiences of daily } \\
\text { living }\end{array}$ & 0.65 & $<0.0001$ \\
\hline MDS-UPDRS Part III - Motor examination & 0.46 & $<0.0001$ \\
\hline MDS-UPDRS Part IV - Motor complications & 0.22 & 0.0003 \\
\hline
\end{tabular}

Abbrevations: PDQ-39, The Parkinson's Disease Questionnaire; SI, summary index score; HY, Hoehn and Yahr stage; SE-ADL, Schwab and England Activities of Daily Living Scale; MMSE, Mini Mental State Examination; BDI, Beck Depression Inventory; MDS-UPDRS, Movement Disorders Society Unified Parkinson's Disease Rating Scale; ICDs, Impulse control disorders

predictors and depression the most significant predictor of HRQoL.

\section{Discussion}

The purpose of this study was to investigate factors that may contribute to low HRQoL in persons with PD. We found that the main clinical determinants of low HRQoL in persons with $\mathrm{PD}$ were depression and the high 
Table 5 Predictors of HRQOL in stepwise multiple regression

\begin{tabular}{|c|c|c|c|}
\hline \multirow[t]{2}{*}{ Model } & \multicolumn{2}{|c|}{ Unstandardized coefficients } & \multirow[t]{2}{*}{$\mathrm{p}$ value } \\
\hline & B & SE & \\
\hline BDI & 0.734 & 0.089 & 0.0001 \\
\hline MDS-UPDRS Part IB & 0.603 & 0.201 & 0.003 \\
\hline MDS-UPDRS Part II & 0.546 & 0.127 & 0.0001 \\
\hline MDS-UPDRS Part III & 0.067 & 0.050 & 0.182 \\
\hline Age & -0.124 & 0.077 & 0.108 \\
\hline Duration of the disease & 0.251 & 0.184 & 0.175 \\
\hline \multirow[t]{3}{*}{ Duration of levodopa treatment } & -0.339 & 0.211 & 0.109 \\
\hline & Multiple & $R^{2}=0.611$ & \\
\hline & Adjusted & $R^{2}=0.599$ & \\
\hline
\end{tabular}

$\mathrm{HRQ}$, health-related quality of life; $\mathrm{SE}$, standard error; $\mathrm{BDI}$, Beck depression inventory; MDS-UPDRS, Movement Disorder Society Unified Parkinson's Disease Rating Scale

burdens of motor and non-motor aspects of daily living. None of the investigated socio-demographic variables significantly associated with HRQoL.

Depression has an average prevalence of about $40 \%$ among persons with PD [20] and is a recognized predictor of low HRQoL. As previous studies [5, 8], we found depression to be one of the most significant determinant of HRQoL. A study by Leentjens et al. [21] demonstrated that the risk factors in the general population for depression-such as older age, female gender, somatic comorbidities, and personal and family history of depression-also predict depression in persons with PD. In our sample the prevalence of depression in female patients was significantly higher than in male patients (58 \% and $43 \%$ respectively, $\mathrm{p}=0.0189$ ). In addition to BDI, depression was more often reported by women according to Part IA of the MDS-UPDRS. Studies examining gender differences regarding prevalence of depression have yielded inconsistent results. While van der Hoek et al. [22] found no difference in the prevalence of depression in male and female persons with PD, Solla et al. [23] found prevalence of depression significantly higher in female persons with PD. The reason why women are more disposed to depression might partly be explained by the greater exposure of competing risk factors, as suggested by Sonnenberg et al. [24] who demonstrated this association in a study on gender differences for depression in the elderly of a general population: controlling for age, comorbid somatic disease, and a number of other risk factors reduced the relative risk of depression in women by more than a half. The female patients in our study were slightly older than the male patients, were more often widowed and had a higher mean number of comorbidities. Men in our cohort were significantly more often married and lived together with their wife and or children. Based on the studies by Sonnenberg et al., it could be assumed that controlling for several factors associated with social and health status might reduce the relative risk of depression in female persons with PD.

In recent years there has been increasing evidence suggesting that the impact of non-motor symptoms on HRQoL is more important than the impact of motor features $[8,9]$. Similar to previous studies, our study revealed that $H R Q o L$ was significantly lower with higher loads of non-motor symptoms. All non-motor symptoms correlated significantly with PDQ-39 SI, except the features of ICDs: a heterogeneous group of pathological behaviours associated with dopamine replacement treatment that include pathological gambling, compulsive sexual behaviour, compulsive shopping, and binge eating, together with pounding and the addiction-like compulsive use of dopamine replacement therapy [25]. A case study by Voon et al. indicated that patients with ICDs have more depressive symptoms [26]. We did not find a statistically significant difference between mean BDI scores for persons with PD and with ICDs (based on positive item 1.6 in MDS-UPDRS Part IA), and persons with PD and without ICDs. Reducing the dosage or discontinuing the administration of dopaminergic agonists or substituting another drug from this group is a frequently effective therapeutic measure [25, 27]. Only a few studies on the impact of ICDs on HRQoL have been published and some found controversial results, i.e. ICDs as a predictor of lower HRQoL [28, 29], but in other studies [30] ICDs did not affect HRQoL. The current study did not reveal a negative impact of ICDs on quality of life. Our finding is supported by a study by Ondo, where only $18 \%$ of patients with increased impulsivity felt that the change was deleterious [30]. Our daily clinical practice suggests that ICDs cause considerable distress to patients' families or caregivers, but the patients themselves are not that annoyed by these behaviours. Out of the 268 patients screened in our study, $7.8 \%$ $(n=21)$ complained of ICDs. It could be assumed that at least one reason why ICDs did not correlate significantly with HRQoL is that the pathological behaviours were relatively mild. About half $(52 \%)$ of these patients reported only slight problems with behavioural disturbances and it could be that the problems did not yet affect their social and occupational functioning.

Dependency in activities of daily living as assessed using the SE-ADL scale and disability in the performance of daily living experiences as measured by the previous version of the UPDRS Part II, have been found to be significant contributors to HRQoL [5]. In a recently published study by Rodriguez-Blazquez et al. [31], who used the MDS-UPDRS Part II, a significant negative association between the more advanced disability and 
HRQoL occurred. The current study demonstrated that disability evaluated by SE-ADL and MDS-UPDRS Part II significantly contributed to lower HRQoL. However, motor symptoms (MDS-UPDRS Part III) were not an independent predictor of HRQoL. Even though for antiparkinsonian treatment Part III with motor assessments is of high importance, it can be seen that for HRQoL, the self-assessment of non-motor symptoms and ability to perform daily activities have more impact.

An axial impairment has been shown to be associated with reduced HRQoL in persons with PD [6]. We found that patients with postural instability and gait disorder dominance had significantly lower overall HRQoL compared to patients with tremor dominance or hypokineticrigid dominance; however, in multiple regression analysis it did not appear to be an independent predictor of HRQoL. Neither was HY stage found to be an independent predictor, though patients with more severe PD $(\mathrm{HY} \geq 3)$ had lower HRQoL than patients with milder PD. The results of the multiple regression analysis were concordant, as higher HY stages reflected an axial involvement with balance and gate impairments. Persons with PD in general, but particularly in patients with the postural instability and gait disorder, are likely to become less able to move around inside their homes and out in the community. They also become more prone to falls as the disease progresses. Regular physical exercise is associated with higher HRQoL, mobility, physical function, slowing the progression of the disease, lower caregiver burden, and less cognitive decline [32]. In view of this, patients with the postural instability and gait disorder may benefit most from education on using rehabilitation activities (e.g. physical exercise, physiotherapy) and assistive devices (e.g. reaching aids). Therefore, in addition to pharmacological treatment, the management of PD should include rehabilitative care, which helps to maintain patients' ability to participate in daily living activities and avoid the decline of HRQoL.

In our study socio-demographic characteristics including age, gender, urban/rural living, level of education, marital status and living alone/with others, did not significantly affect overall HRQoL. However, we found several associations between these factors and domains of PDQ-39. Women received lower scores for emotional well-being and bodily discomfort, which is in accordance with the study by Carod-Artal et al. [5]. As regards to patients' living status, those who lived alone received lower scores for emotional well-being compared with patients who lived with others. Another study by Winter et al. showed that patients living alone had worse HRQoL than patients living with somebody [7]. Our results did not reveal any statistically significant difference in HRQoL among patients with different educational backgrounds, whereas greater number of years in education was found to be associated with higher HRQoL in a study by Carod-Artal et al. [5]. Klepac et al. [33] found that rural patients had lower overall HRQoL, with most of the domains of PDQ-39 affected. In contrast, the results of our study suggest that living area is not associated with overall HRQoL. Moreover, our results showed that patients living in rural areas had significantly less stigmatization and better social support than patients living in urban areas.

The study has some methodological limitations that ought to be recognized and taken into account when interpreting the findings. The analysis was based on clinical data collected at a single point in time; therefore, any pattern of progression of the disease could not be estimated. Also, we cannot exclude the possibility that some of the variables, such as gender (female patients outnumbered male patients) or stage of disease $(62.6 \%$ of patients had $\mathrm{HY} \geq 3$ ) could have influenced the results found.

The strength of the study was the relatively large sample of persons with $\mathrm{PD}$, which included institutionalised and severely ill patients, and thus was representative of the PD population as a whole. The study participants were also evaluated with a wide range of clinimetric properties that covered motor, non-motor, functional, cognitive and emotional aspects.

\section{Conclusion}

The results of our study suggest that both non-motor and motor aspects affect the daily living and HRQoL of persons with PD. Our results are consistent with other studies that have suggested depression to be the strongest predictor of HRQoL in persons with PD and therefore depression should be recognized early and treated appropriately. Attention should also be paid to other common non-motor symptoms that may require a multidisciplinary approach, such as cognitive impairment, sleep problems, urinary problems and fatigue. A novel observation of this study was that ICDs were the only non-motor symptom not associated with lower HRQoL. Further research in this setting is planned to explore the role of behavioural disturbances in HRQoL among persons with PD.

\section{Abbreviations}

BDI: Beck Depression Inventory; HRQoL: Health-Related Quality of Life; HY: Hoehn and Yahr scale; ICDs: Impulse Control Disorders; MDS-UPDRS: Movement

Disorders Society Unified Parkinson's Disease Rating Scale; MMSE: Mini Mental State Examination; PD: Parkinson's Disease; PDQ-39: Parkinson's Disease Quality of Life Questionnaire; SE-ADL: Schwab and England Activities of Daily Living scale; SI: Summary Index.

\section{Competing interests}

The authors declare that they have no competing interests. 


\section{Authors' contributions}

LKE collected data, interpreted the results, and drafted the whole manuscript. MR performed the statistical analysis and contributed in interpretation of data. TP and MM contributed to the acquisition of data. PT was involved in the study design, data collection, drafting the manuscript, and gave the final approval of the version to be published. All authors read and approved the final manuscript

\section{Acknowledgements}

This study was supported by the Grant 3.2.1001.11-0017 of the EU European Regional Development Fund and the Grant IUT2-4 of the Estonian Research Council.

Received: 19 December 2014 Accepted: 6 June 2015

Published online: 20 June 2015

\section{References}

1. Taba P, Asser T. Epidemiology of Parkinson's disease. Rev Clin Gerontol 2004;14:211-28.

2. $\quad$ Lees AJ, Hardy J, Revesz T. Parkinson's disease. Lancet. 2009;373:2055-66.

3. Chaudhuri KR, Odin P, Antonini A, Martinez-Martin P. Parkinson's disease: the non-motor issues. Parkinsonism Relat Disord. 2011;17:717-23.

4. Poewe W, Mahlknecht P. The clinical progression of Parkinson's disease. Parkinsonism Relat Disord. 2009;15 Suppl 4:S28-32.

5. Carod-Artal FJ, Vargas AP, Martinez-Martin P. Determinants of quality of life in Brazilian patients with Parkinson's disease. Mov Disord. 2007;22:1408-15.

6. Muslimovic D, Post B, Speelman JD, Schmand B, de Haan RJ. Determinants of disability and quality of life in mild to moderate Parkinson disease. Neurology. 2008;70:2241-7.

7. Winter Y, von Campenhausen S, Popov G, Reese JP, Balzer-Geldsetzer M, Kukshina $A$, et al. Social and clinical determinants of quality of life in Parkinson's disease in a Russian cohort study. Parkinsonism Relat Disord. 2010;16:243-8

8. Gallagher DA, Lees AJ, Schrag A. What are the most important nonmotor symptoms in patients with Parkinson's disease and are we missing them? Mov Disord. 2010;25:2493-500.

9. Martinez-Martin P, Rodriguez-Blazquez C, Kurtis MM, Chaudhuri KR. The impact of non-motor symptoms on health-related quality of life of patients with Parkinson's disease. Mov Disord. 2011;26:399-406.

10. Goetz CG, Tilley BC, Shaftman SR, Stebbins GT, Fahn S, Martinez-Martin P, et al. Movement Disorder Society-sponsored revision of the Unified Parkinson's Disease Rating Scale (MDS-UPDRS): scale presentation and clinimetric testing results. Mov Disord. 2008;23:2129-70.

11. Gibb WR, Lees AJ. The relevance of the Lewy body to the pathogenesis of idiopathic Parkinson's disease. J Neurol Neurosurg Psychiatry. 1988;51:745-52.

12. Peto $V$, Jenkinson $C$, Fitzpatrick $R$, Greenhall $R$. The development and validation of a short measure of functioning and well being for individuals with Parkinson's disease. Qual Life Res. 1995:4:241-8.

13. Krikmann U, Taba P, Lai T, Asser T. Validation of an Estonian version of the Parkinson's Disease Questionnaire (PDQ-39). Health Qual Life Outcomes. 2008. doi:10.1186/1477-7525-6-23.

14. Hoehn MM, Yahr MD. Parkinsonism: onset, progression and mortality. Neurology. 1967;17:427-42.

15. Schwab RS, England AC. Projection technique for evaluating surgery in Parkinson's disease. In: Gillingham FJ, Danoldson IML, editors. Third symposium on Parkinson's disease. Edinburgh: Churchill Livingstone; 1969. p. 152-7.

16. Beck AT, Ward CH, Mendelson M, Mock J, Erbaugh J. An inventory for measuring depression. Arch Gen Psychiatry. 1961:4:561-71.

17. Leentjens AF, Verhey FR, Luijckx GJ, Troost J. The validity of the beck depression inventory as a screening and diagnostic instrument for depression in patients with Parkinson's disease. Mov Disord. 2000;15:1221-4.

18. Folstein MF, Folstein SE, McHugh PR. "Mini-mental state". a practical method for grading the cognitive state of patients for the clinician. J Psychiatr Res. 1975;12:189-98.

19. Jenkinson C, Fitzpatrick R, Peto V, Greenhall R, Hyman N. The Parkinson's Disease Questionnaire (PDQ-39): development and validation of a Parkinson's disease Summary Index Score. Age Ageing. 1997:26:353-7.

20. Slaughter JR, Slaughter KA, Nichols D, Holmes SE, Martens MP. Prevalence, clinical manifestations, etiology, and treatment of depression in Parkinson's disease. J Neuropsychiatry Clin Neurosci. 2001;13:187-96.
21. Leentjens AF, Lousberg R, Verhey FR. Markers for depression in Parkinson's disease. Acta Psychiatr Scand. 2002;106:196-201.

22. van der Hoek TC, Bus BA, Matui P, van der Marck MA, Esselink RA, Tendolkar I. Prevalence of depression in Parkinson's disease: effects of disease stage, motor subtype and gender. J Neurol Sci. 2011;310:220-4.

23. Solla P, Cannas A, Ibba FC, Loi F, Corona M, Orofino G, et al. Gender differences in motor and non-motor symptoms among Sardinian patients with Parkinson's disease. J Neurol Sci. 2012;323:33-9.

24. Sonnenberg CM, Beekman AT, Deeg DJ, van Tilburg W. Sex differences in late-life depression. Acta Psychiatr Scand. 2000;101:286-92.

25. Djamshidian A, Averbeck BB, Lees AJ, O'Sullivan SS. Clinical aspects of impulsive compulsive behaviours in Parkinson's disease. J Neurol Sci. 2011;310:183-8.

26. Voon $V$, Sohr M, Lang AE, Potenza MN, Siderowf AD, Whetteckey J, et al. Impulse control disorders in Parkinson's disease: a multicenter case-contro study. Ann Neurol. 2011;69:986-96.

27. Giovannoni G, O'Sullivan JD, Turner K, Manson AJ, Lees AJL. Hedonistic homeostatic dysregulation in patients with Parkinson's disease on dopamine replacement therapies. J Neurol Neurosurg Psychiatry. 2000:68:423-8.

28. Phu AL, Xu Z, Brakoulias V, Mahant N, Fung VS, Moore GD, et al. Effect of impulse control disorders on disability and quality of life in Parkinson's disease patients. J Clin Neurosci. 2014;21:63-6.

29. Leroi I, Ahearn DJ, Andrews M, McDonald KR, Byrne EJ, Burns J. Behavioural disorders, disability and quality of life in Parkinson's disease. Age Ageing. 2011. doi:10.1093/ageing/afr07826.

30. Ondo WG, Lai D. Predictors of impulsivity and reward seeking behavior with dopamine agonists. Parkinsonism Relat Disord. 2008;14:28-32.

31. Rodriguez-Blazquez C, Rojo-Abuin JM, Alvarez-Sanchez M, Arakaki T, Bergareche-Yarza A, Chade A, et al. The MDS-UPDRS Part II (motor experiences of daily living) resulted useful for assessment of disability in Parkinson's disease. Parkinsonism Relat Disord. 2013;19:889-93.

32. Oguh $O$, Eisenstein $A$, Kwasny M, Simuni T. Back to the basics: regular exercise matters in Parkinson's disease: results from the national Parkinson foundation QII registry study. Parkinsonism Relat Disord. 2014:20:1221-5.

33. Klepac N, Pikija S, Kraljic T, Relja M, Trkulja V, Juren S, et al. Association of rural life setting and poorer quality of life in Parkinson's disease patients: a cross-sectional study in Croatia. Eur J Neurol. 2007;14:194-8.

\section{Submit your next manuscript to BioMed Central and take full advantage of:}

- Convenient online submission

- Thorough peer review

- No space constraints or color figure charges

- Immediate publication on acceptance

- Inclusion in PubMed, CAS, Scopus and Google Scholar

- Research which is freely available for redistribution 